\title{
Endogenous cross-talk of fungal metabolites
}

\section{Kevin J. Sheridan, Stephen K. Dolan and Sean Doyle*}

Department of Biology, Maynooth University, Maynooth, Ireland

\section{Edited by:}

Nancy Keller, University of

Wisconsin-Madison, USA

\section{Reviewed by:}

Philipp Wiemann, University of

Wisconsin-Madison, USA

Daniel Panaccione, West Virginia

University, USA

\section{*Correspondence:}

Sean Doyle, Department of Biology,

Maynooth University, Maynooth,

Kildare, Ireland

e-mail: sean.doyle@nuim.ie

\begin{abstract}
Non-ribosomal peptide (NRP) synthesis in fungi requires a ready supply of proteogenic and non-proteogenic amino acids which are subsequently incorporated into the nascent NRP via a thiotemplate mechanism catalyzed by NRP synthetases. Substrate amino acids can be modified prior to or during incorporation into the NRP, or following incorporation into an early stage amino acid-containing biosynthetic intermediate. These post-incorporation modifications involve a range of additional enzymatic activities including but not exclusively, monooxygenases, methyltransferases, epimerases, oxidoreductases, and glutathione S-transferases which are essential to effect biosynthesis of the final NRP. Likewise, polyketide biosynthesis is directly by polyketide synthase megaenzymes and cluster-encoded ancillary decorating enzymes. Additionally, a suite of additional primary metabolites, for example: coenzyme A (CoA), acetyl CoA, S-adenosylmethionine, glutathione (GSH), $\mathrm{NADPH}$, malonyl CoA, and molecular oxygen, amongst others are required for NRP and polyketide synthesis (PKS). Clearly these processes must involve exquisite orchestration to facilitate the simultaneous biosynthesis of different types of NRPs, polyketides, and related metabolites requiring identical or similar biosynthetic precursors or co-factors. Moreover, the near identical structures of many natural products within a given family (e.g., ergot alkaloids), along with localization to similar regions within fungi (e.g., conidia) suggests that cross-talk may exist, in terms of biosynthesis and functionality. Finally, we speculate if certain biosynthetic steps involved in NRP and PKS play a role in cellular protection or environmental adaptation, and wonder if these enzymatic reactions are of equivalent importance to the actual biosynthesis of the final metabolite.
\end{abstract}

Keywords: NRPS, gliotoxin, natural products, secondary metabolites, redox homeostasis, siderophores, systems biology, PKS

\section{INTRODUCTION}

Non-ribosomal peptides (NRP) and polyketides are produced by both fungi and bacteria, via non-ribosomal peptide synthesis (NRPS) and polyketide synthesis (PKS), respectively, and are often associated with aiding the organisms to adapt to, or survive in, a hostile environment such as the presence of competing microorganisms, nutrient limitation, and protection against insect immune systems (Meier and Burkart, 2009; Wiemann and Keller, 2014). Although many NRPs and polyketides have known functions [e.g., siderophores (iron acquisition) and antibiotics (bacterial inhibition)] and biomedical applications (e.g., cyclosporin, lovastatin, and mycophenolic acid), the true biological function of many NRPs and polyketides identified to date is unknown, and abrogation of the biosynthesis of certain NRPs, or polyketides, may be without major consequence for the organism (O'Hanlon et al., 2011; Hansen et al., 2012; Steinchen et al., 2013).

With respect to the actual processes, NRPS in fungi is mediated by a combination of large multi-functional enzymes known as NRP synthetases, which include adenylation, thiolation, condensation, and sometimes tailoring domains. NRP synthetases require post-translational modification via $4^{\prime}$-phosphopantetheinylation, mediated by $4^{\prime}$-phosphopantetheinyl transferase (4'-PPTase), to yield the active holo-NRP synthetase. This modification, which requires coenzyme $\mathrm{A}(\mathrm{CoA})$ as the $4^{\prime}$-phosphopantetheine source, requires that each thiolation domain within an NRP synthetase is modified at a specific serine residue (Stack et al., 2007). In addition, so-called decorating enzymes or domains are responsible for the modification of NRP biosynthetic intermediates, which may be either tethered to, or released from, the NRP synthetase during the modification process. Likewise, polyketides are biosynthesized from acyl CoA precursors (e.g., malonyl CoA) by multi-modular enzymes consisting of essential ketosynthase, acyl carrier protein and acyltransferase, amongst other, domains (Weissman, 2014). As shown for Streptomyces spp. intracellular ATP levels may also affect NRP production in fungi (Li et al., 2008). Unlike PK synthases, NRP synthetases require ATP for NRPS.

Both proteogenic and non-proteogenic amino acids (e.g., ornithine) may be essential precursor substrates for NRP formation and during NRPS are conjugated together generally via peptide bond formation. Moreover, substrate amino acids can be modified either prior to, or during, NRPS (Thykaer and Nielsen, 2003; Chocklett and Sobrado, 2010). In addition, many other cellular components more commonly associated with primary metabolism are required for NRPS and PKS. Amongst others, these include S-adenosylmethionine (SAM), isoprenyl, and mevalonyl moieties, nicotinamide adenine dinucleotide phosphate (NADPH), glutathione (GSH), malonyl CoA, and acetyl CoA (Davis etal., 2011; Scharf etal., 2011; Haas, 
2012; Yasmin etal., 2012). This suggests significant interplay between what is currently considered to be primary and secondary metabolism and that essential re-consideration must be given to the integration of these two historically defined discrete systems.

In fungi, the biosynthesis of a specific NRP is generally encoded by genes located within a gene cluster, which are often located in the sub-telomeric regions of chromosomes $(\mathrm{McD}$ onagh et al., 2008). The activity of these secondary metabolite (SM) gene clusters is controlled by local chromatin structure, which is effected via histone post-translational modification (e.g., methylation or acetylation). The influence of histone modifying enzymes on SM production was first reported for sterigmatocystin (ST) production and gene regulation in A. nidulans. The authors uncovered that histone deacetylase mutant $\Delta h d a A$ bypassed the requirement for the general SM activator LaeA, resulting in overproduction of the subtelomeric metabolites ST and penicillin (Shwab et al., 2007). In the endophytic filamentous fungus Epichloë festucae the gene clusters responsible for the production of bioprotective lolitrems and ergot alkaloids were shown to be derepressed following deletion of the genes encoding either the H3K9- (ClrD) or H3K27- (EzhB) histone methyltransferases. A further enhancement of cluster expression was evident when both of these methyltransferases were deleted (Chujo and Scott, 2014). Indeed, the production of many SM in Aspergillus spp. is controlled by a methyltransferase, laeA, and orthologs of this gene are found in many fungi (Bok and Keller, 2004). Interestingly, LaeA has been shown to counteract the establishment of heterochromatic marks thus activating SM production. Deletion of laeA in A. nidulans resulted in highly elevated $\mathrm{H} 3 \mathrm{~K} 9$ methylation levels suggesting that LaeA counteracts H3K9 trimethylation and thus heterochromatin formation at the ST locus (Reyes-Dominguez et al., 2010). The Velvet complex, which is comprised of the light-dependent regulators $\mathrm{VeA}$ and VelB, also regulates SM production, and serves to coordinate SM production and fungal development. It is now clear that the VelB-VeA-LaeA complex coordinates fungal sexual development and secondary metabolism (Bayram et al., 2008).

Thus, it appears that a truly phenomenal degree of cross-talk exists between so-called primary and secondary metabolism, also involving genetic regulation, to facilitate NRP and polyketide, and indeed other SM, production (Figure 1). Primary metabolism is required to provide the essential biosynthetic precursors for NRPS, many NRPs share substrate amino acids and additional co-substrates, chromatin structure apparently controls much SM gene cluster expression, and evidence is also emerging that NRPS pathways interact such that alterations in the biosynthesis of specific NRPs may impact on the production of apparently unrelated metabolites (O'Keeffe et al., 2014; Wiemann et al., 2014). This review is intended to highlight some of the metabolite cross-talk which has been identified to date, and to suggest possible future directions which may further illuminate our understanding of this complex molecular vista- which may, in part, necessitate the development of new models to augment current paradigms of distinct levels of cellular metabolism.

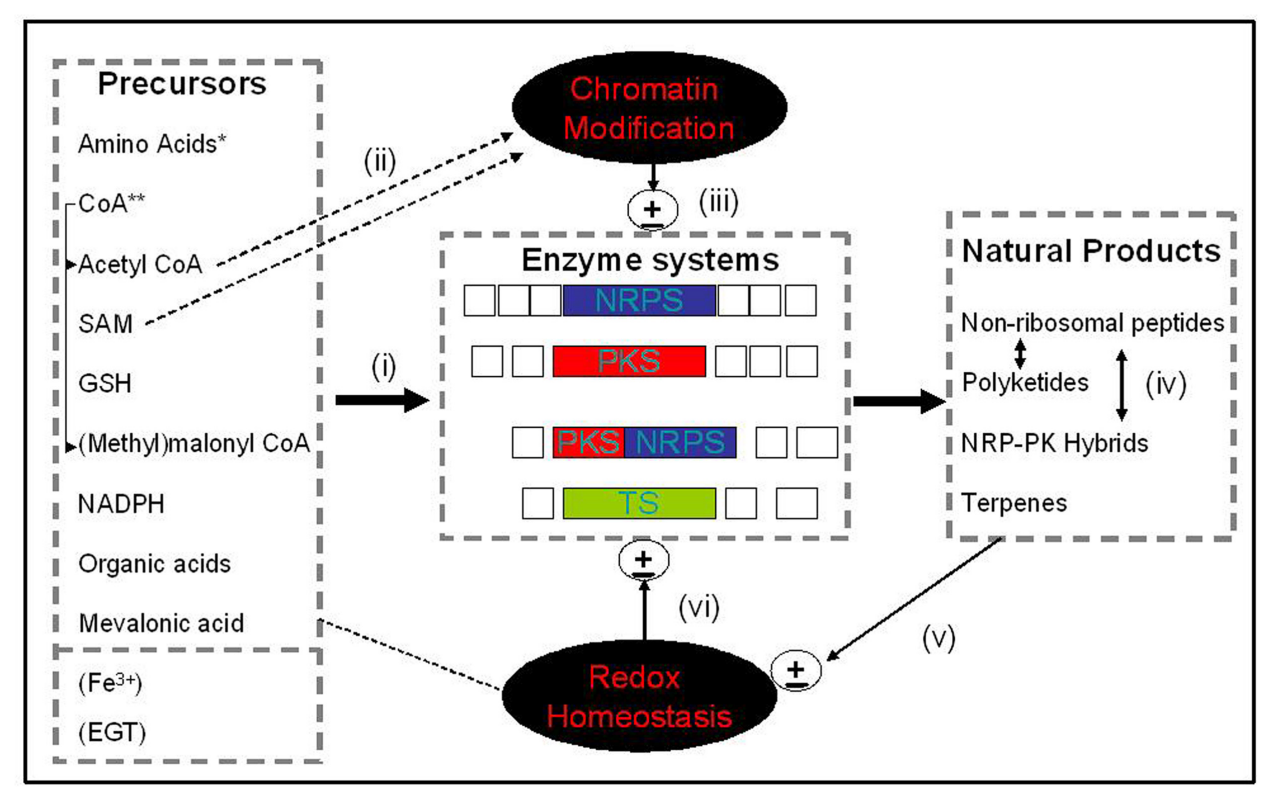

FIGURE 1 | Systems overview. An integrated depiction of how precursors from 'primary metabolism' (i) feed into enzyme systems [NRPS, PKS, NRPS-PKS, and terpene synthases (TS)] which effect natural product/secondary metabolite biosynthesis and, in part, (ii) affect both chromatin structure (e.g., Acetyl CoA and SAM), and cellular redox status (e.g., GSH, EGT, and $\mathrm{Fe}^{3+}$ ). Chromatin structure, in turn, can (iii) regulate gene cluster expression and metabolite biosynthesis. (iv) Resultant natural products can interact synergistically to enable cellular function (e.g., iron uptake, antioxidant adaptation), or (v) influence cellular redox homeostasis which in turn can (vi) impact on gene cluster expression. Note: $\mathrm{Fe}^{3+}$ and EGT are not 'precursors' per se, however, they either bind to (e.g., $\mathrm{Fe}^{3+}$-siderophores), or their biosynthesis is influenced by, natural products. *amino acids, proteogenic or non-proteogenic; ${ }^{*} \mathrm{CoA}$, Coenzyme $A$, a substrate or co-factor for natural product biosynthesis; EGT, Ergothioneine; Open boxes, ancillary enzymes involved in PKS, NRPS, or TS. 


\section{PRIMARY METABOLITE INVOLVEMENT IN SM BIOSYNTHESIS \\ COA AND ACETYL CoA}

It is essential to consider the sources of primary metabolites used during fungal NRPS and PKS in order to begin to see why there may be interactions between apparently independent biosynthetic pathways (Figure 1). CoA is somewhat unique, as it functions (i) as a direct substrate which facilitates NRP synthetase and PK synthase activation via $4^{\prime}$-PPTase, and (ii) as malonyl CoA in PKS, and, as acetyl CoA, as the source of acetyl groups for NRPS and histone modification (Stack et al., 2009; Jeon and Lee, 2014; Weissman, 2014). To enable CoA biosynthesis, L-valine and L-aspartate are initially converted to pantoate and $\beta$-alanine, respectively, followed by condensation to form pantothenate (vitamin B5), which in turn is converted to CoA by the action of a multi-step biosynthetic pathway involving condensation to ADP (Spry et al., 2008; Chen et al., 2014a; Figure 1). Cytosolic acetyl CoA is provided by $\beta$-oxidation of fatty acids, from acetate through the action of acetate synthetase (Acs1), and via the ATP-citrate lyase (Acl1) which uses citrate as a substrate (Griffiths et al., 2012). Indeed it has been demonstrated that Acl1 and 2 function to regulate cytosolic acetyl CoA levels in A. niger which in turn affects growth and development (Chen et al., 2014a). Thus, it is clear that provision of two essential biomolecules (CoA and acetyl CoA) for fungal NRPS and PKS requires close interplay with primary metabolic processes. Interestingly, although studies directly pertaining to NRPs appear rare, it has been demonstrated that biosynthesis of the polyketide lovastatin in A. terreus is enhanced by media supplementation with calcium D-pantothenate, amongst other B vitamins (Bizukojc et al., 2007).

\section{SAM}

$S$-adenosylmethionine is the major source of methyl groups for cellular reactions involving methylation, and is directly synthesized via the action of SAM synthetase (Sauter et al., 2013). In fungi, the methionine cycle enables SAM biosynthesis, whereby L-homocysteine is converted to L-methionine via methionine synthase, SAM is formed in turn via SAM synthetase which requires ATP (Sieńko et al., 2009). Cellular methylation reactions (e.g., NRPS and PKS) then consume SAM to produce $S$-adenosylhomocysteine (SAH), which in turn is converted to L-homocysteine, thereby completing the methionine cycle (Liao et al., 2012). In A. nidulans, a SAM synthetase, SasA, has been demonstrated to affect SAM availability which is important for the production of ST, but also may play a role in coordinating fungal secondary metabolism and development (Gerke et al., 2012). These authors demonstrated SasA interaction with histone $2 \mathrm{~B}$ via TAP-tag technology and LC-MS, and suggested that this interplay may facilitate epigenetic control via methylation. SAM availability is essential for the biosynthesis of a range of polyketides and NRPs including gliotoxin, where it acts as a methyl source for the $N$-methyltransferase GliN (Scharf et al., 2014), and the negative regulator of gliotoxin biosynthesis GtmA, which attenuates gliotoxin biosynthesis via SAM-dependent bisthiomethylation of dithiolgliotoxin (Dolan et al., 2014). SAM is also the provider of methyl groups for the biosynthesis of many other NRPs and polyketides such as butyrolactone III
(Guo etal., 2013a). Forseth etal. (2011) identified a plethora of methylated biosynthetic intermediates/shunt metabolites associated with gliotoxin biosynthesis which allowed the authors to speculate about the complex reactions required within this metabolic pathway.

Methionine and SAM are sulfur-containing metabolites, and it has recently been demonstrated that the bZIP transcription factor MetR plays an important role in sulfur assimilation in A. fumigatus (Amich et al., 2013). While these authors elegantly demonstrated that MetR functionality was essential for growth on inorganic sulfur sources, it was observed to be less important for regulating genes involved in methionine metabolism. Nonetheless, they identified a key interaction with iron homeostasis, whereby under sulfur-limiting conditions, genes involved in siderophore biosynthesis (sidA), siderophore transport (mirB), and iron regulation (hapX) underwent increased expression in A. fumigatus $\Delta$ metR although sufficient $\mathrm{Fe}^{3+}$ was available in the media. Moreover, significantly elevated levels of the intracellular siderophore, ferricrocin, a NRP, were also observed which was necessary to chelate elevated intracellular $\mathrm{Fe}^{3+}$ to prevent cellular damage in $\Delta m e t R$. Given the essential role of Fe-S cluster proteins in energy generation, this regulatory cross-talk, which involved altered iron-sensing due to defective sulfur assimilation, and led to altered NRP [ferricrocin (FC)] levels significantly underpins the integrated nature of cellular metabolism in fungi.

\section{GSH}

The biosynthesis of gliotoxin, acetylaranotin, and related epipolythiodioxopiperazine (ETP) compounds requires biosynthetic intermediate sulfurization and it has been established that GSH is this sulfur source (Davis et al., 2011; Scharf et al., 2011; Guo et al., 2013b). Furthermore, Atanasova et al. (2013) have noted elevated expression of genes predicted to be involved in GSH formation, possibly as a precursor to enable the biosynthesis of gliotoxin in Trichoderma virens. In any case, during gliotoxin biosynthesis a gene cluster-encoded glutathione $S$-transferase (GST) mediates GSH conjugation to a highly reactive acyl imine intermediate which results in a bis-glutathionylated product, which is subsequently processed by a suite of enzymes, initially by a $\gamma$-glutamyl cyclotransferase (GliK in the case of gliotoxin biosynthesis) to the final product (Davis et al., 2011; Gallagher et al., 2012; Scharf et al., 2011, 2013). Thus, a key cellular reductant, which can also undergo oxidation to the GSSG form to maintain cellular redox homeostasis, is an essential substrate for NRP biosynthesis. Indeed, it is interesting to speculate how, and why, fungi utilize an antioxidant (GSH) to generate an alternative metabolite, gliotoxin, with redox-active properties. Perhaps the ability of fungi to effect gliotoxin (and related ETP) but not GSH secretion is in part responsible for this apparent puzzling situation.

\section{ERGOTHIONEINE}

Ergothioneine (EGT) is a sulfurized and tri- $\mathrm{N}$-methylated L-histidine derivative, and functions as an antioxidant due to its high redox potential (Fahey, 2013). It is produced by non-yeast fungi, with the gene EGT-1 found in all fungal phyla, except the Saccharomycotina subphylum (Genghof, 1970; Jones et al., 2014). 
EGT exists as a tautomer between its thione and thiol forms, however, it is predominantly found in the thione form at physiological pH (Carlsson et al., 1974). While most naturally occurring thiols have a redox potential ranging from $-0.2 \mathrm{~V}$ and $-0.32 \mathrm{~V}$, EGT has a redox potential of $-0.06 \mathrm{~V}$, which means that it is less susceptible to auto-oxidation compared to GSH (Jocelyn, 1972). In Neurospora crassa, ergothioneine has been proposed to protect conidia against oxidative stress (Bello et al., 2012). Although not strictly a NRP, recent work has revealed further requirements for provision of SAM and $\gamma$-glutamylcysteine (and molecular $\mathrm{O}_{2}$ ) to enable ergothioneine biosynthesis in Mycobacteria (Seebeck, 2010). In A. fumigatus, Gallagher et al. (2012) observed that EGT levels increased significantly when production of the NRP gliotoxin was abrogated. The significance of this correlation, if any, has yet to be elucidated. In other fungi (Schizosaccharomyces pombe), the multi-functional enzyme Egt-1, which comprises a methyltransferase domain and a hercynylcysteine sulfoxide synthase activity, requires both SAM and L-cysteine to enable ergothioneine biosynthesis - whereby SAM provides methyl groups and L-cysteine is the sulfur source (Pluskal et al., 2014). As noted, Gallagher etal. (2012) were the first to demonstrate that deletion of gliK from A. fumigatus not only abrogated gliotoxin biosynthesis, but also resulted in significantly elevated levels of intracellular ergothioneine. Although these authors noted a significantly elevated sensitivity to $\mathrm{H}_{2} \mathrm{O}_{2}$-induced oxidative stress, the precise mechanistic details of the interaction between these redox-active metabolites is presently unclear, and future work is likely to dissect the nature of this cross-talk. However, it is not inconceivable that ergothioneine biosynthesis is increased to, in part, compensate for the abrogation of gliotoxin biosynthesis at a specific biosynthetic step.

\section{NAPDH}

Nicotinamide adenine dinucleotide phosphate requirement as a reductant in NRPS and PKS is well-established (Manavalan et al., 2010; Wilson etal., 2013) and recent work in mammalian cell lines (Fan etal., 2014) has demonstrated that both the oxidative pentose phosphate pathway and one-carbon metabolism are important cellular sources of NADPH. While it has been reported that metabolic engineering of the pentose phosphate pathway consequent to increased abundance of 6-phosphogluconate dehydrogenase - can lead to enhanced cellular NADPH availability in $A$. niger (R Poulsen et al., 2005), to our knowledge little assessment of alternative sources of NADPH in fungi has been forthcoming. Thus, in future assessments of integrated fungal metabolism, it is possible that relevant interactions between NRPS, PKS, and availability of alternative reducing power require evaluation.

\section{AMINO ACIDS}

A link between amino acid biosynthesis and secondary metabolism has been described in the ascomycetous plant pathogen Leptosphaeria maculans. The NRP sirodesmin PL, an ETP, which is the causal agent of blackleg disease of Brassica napus, has been shown to be regulated by the cross pathway control gene $c p c A$, which in turn regulates amino acid biosynthesis. During amino acid starvation, amino acids are diverted from sirodesmin biosynthesis into amino acid biosynthesis, and a silenced $c p c A$ mutant was shown to produce significantly higher levels of sirodesmin PL after prolonged amino acid starvation compared to the wildtype strain. However, it has yet to be established if this effect is mediated directly or indirectly through the sirodesmin pathway specific transcriptional factor, sirZ (Elliott et al., 2011). The rice pathogen Fusarium fujikuroi produces the NRP apicidin F under nitrogen-sufficient conditions through the global regulator AreB (Niehaus et al., 2014). Similarly, production of the $F$. fujikuroi $\mathrm{PKS} / \mathrm{NRPS}$-derived mycotoxin fusarin $\mathrm{C}$ is induced by an excess of nitrogen (Wiemann et al., 2010). Organic acids such as fumarate, anthranilic acid, 4-hydroxyphenylpyruvate, and indole3-pyruvate also are incorporated into NRPs in fungi (Wackler et al., 2012; Steinchen et al., 2013; Walsh et al., 2013).

\section{RIBOSOMAL PEPTIDE SYNTHETIC PATHWAY (RiPS)}

The discovery of the biosynthetic mechanism, via a ribosomal peptide synthetic pathway (RiPS), for ustiloxin B in A. flavus by Umemura et al. (2014) can be thought to further contribute to the blurring distinction between primary and secondary metabolism. Ustiloxin B is a modified YAIG tetrapeptide, conjugated to norvaline via L-tyrosine. However, it appears that YAIG is derived from a proteinaceous precursor, followed by extensive enzymatic conversion, which is encoded by a discrete gene cluster, to ustiloxin B. Of special interest is the presence of a $S$ atom in ustiloxin $B$, which is coincident with the presence of a GST, cysteine desulfurase and $\gamma$-glutamyl transpeptidase in the cognate gene cluster (Umemura et al., 2014). Although not noted by the authors, it is tempting to speculate that a similar biochemistry to that which has evolved to enable ETP biosynthesis in fungi, is also operational in the biosynthesis of a RiPS product, ustiloxin B, in an NRPSindependent manner. This work is the first example of RiPS in Ascomycetes.

\section{METABOLITES AND NON-PROTEOGENIC AMINO ACIDS - THE ORNITHINE EXEMPLAR}

Siderophores are NRPs secreted by fungi and bacteria that scavenge free ferric iron $\left(\mathrm{Fe}^{3+}\right)$ in the immediate environment to facilitate its transport into the cell, and this mechanism is deployed by both animal and plant pathogens (Cochliobolus heterostrophus) to acquire iron (Haas, 2012; Zhang et al., 2013). Because $\mathrm{Fe}^{3+}$ is required for primary metabolism, it is clear that these NRPs also act as a nexus between both primary and secondary functionalities within fungi. Since excess intracellular $\mathrm{Fe}^{3+}$ is deleterious, intracellular siderophores also exist to maintain intracellular $\mathrm{Fe}^{3+}$ homeostasis and prevent Fenton reaction oxidative damage (Eisendle etal., 2006). In Aspergillus spp. such as $A$. nidulans and $A$. fumigatus, triacetylfusarinine $\mathrm{C}$ (TAFC) and fusarinine $\mathrm{C}$ (FusC) are secreted to acquire $\mathrm{Fe}^{3+}$, while $\mathrm{FC}$ is the key intracellular siderophore which effects $\mathrm{Fe}^{3+}$ storage. It is notable that in A. niger, TAFC is absent and coprogen B and ferrichrome are the main secreted siderophores, with the latter comprising the main intracellular siderophore (Franken et al., 2014). Biosynthesis of siderophores necessitates interaction between enzyme systems at multiple levels. Firstly, one of the key non-proteinaceous amino acids, L-ornithine, essential for siderophore biosynthesis, must be converted to $N$-hydroxyL-ornithine via the action of ornithine monooxygenase, SidA 
(Schrettl et al., 2004; Hissen et al., 2005). Interestingly, Beckmann etal. (2013) have demonstrated that mitochrondrially derived ornithine is primarily utilized for siderophore biosynthesis - indicating a localization-type cross-talk. These authors also demonstrated metabolite cross-talk between siderophore and polyamine formation, whereby ornithine acts a co-substrate for SidA and ornithine decarboxylase (ODC), the latter enzyme acting to provide biosynthetic precursors for polyamine biosynthesis. Secondly, mevalonate, a biosynthetic intermediate in ergosterol biosynthesis, is also required for siderophore biosynthesis and is enzymatically converted to anhydromevalonyl-CoA to facilitate incorporation into FusC and TAFC (Schrettl et al., 2007). Finally, in A. fumigatus, FusC is converted to TAFC by enzyme-mediated acetylation via the action of SidG in an acetyl CoA-dependent reaction. The cross-talk between ergosterol and siderophore biosynthesis has proven to be especially significant, as it has been demonstrated that inhibition of HMG CoA reductase, the enzyme responsible for mevalonate production (Figure 1), also attenuates siderophore biosynthesis, and virulence in a murine ocular keratitis model system, (Leal et al., 2013). Moreover, this inhibitory effect is enhanced by utilization of iron chelators to further inhibit iron uptake by fungi. Thus, interfering with metabolite cross-talk systems may bring therapeutic benefits.

\section{METABOLITE CROSS-TALK AND OXIDATIVE STRESS}

As aerobic organisms, fungi are subject to oxidative stress due to the production of reactive oxygen species (ROS) as side-products of metabolic pathways. Almost $90 \%$ of ROS in aerobic organisms is produced through the electron transport chain, while oxidases such as NADPH oxidase also contribute toward the ROS content of the cell (Takemoto et al., 2007; Starkov, 2008). Control of ROS levels is important as cellular components can be modified by ROS, particularly enzymes containing [ $\mathrm{Fe}-\mathrm{S}]$-clusters and active thiol groups. Oxidative stress, whereby the ROS concentration rises above tolerable levels, thereby leading to deleterious modification of cellular components, can interfere with cellular metabolism and regulatory pathways (Lushchak, 2011). Enzymes such as catalases, glutathione peroxidases, and superoxide dismutases actively protect against oxidative stress, while fungi also produce antioxidant molecules such as $\alpha$-tocopherols, ascorbic acid, carotene and GSH (Halliwell and Gutteridge, 2007). SM also interacts with ROS in many ways. Some SMs act as antioxidants (EGT, as described above) or otherwise protect against oxidative stress (Bello et al., 2012), while others use oxidative stress as a signal to initiate biosynthesis, while some SMs, like ETPs, enact toxic effects through ROS generation and redox reactions (Gardiner et al., 2005a).

Epipolythiodioxopiperazines are categorized by a signature disulfide bridge (Gardiner et al., 2005a) and gliotoxin is the best studied of the ETPs. It is produced by A. fumigatus, and its biosynthesis is mediated by the products of the gli gene cluster, with the NRP synthetase GliP starting the pathway through the fusion of L-phenylalanine and L-serine (Balibar and Walsh, 2006), while many of the other genes encode decorating enzymes. Gliotoxin toxicity is intrinsically linked to its disulfide bridge, and redox cycling between the disulfide and dithiol forms allows for the production of ROS. The ability of gliotoxin to form mixed disulfide bonds with proteins, resulting in their inactivation, is another example of the redox-dependant action of the disulfide bridge (Waring et al., 1995). A. fumigatus in part detoxifies gliotoxin by closing the disulfide bride via the oxidoreductase GliT, which 'disarms' the toxic effects of the molecule, although a cryptic reductase activity of GliT has also been noted (Scharf et al., 2010; Schrettl et al., 2010). Another ETP, sporidesmin, effects toxicity in a similar manner to gliotoxin (Middleton, 1974; Munday, 1982). The redox activity of gliotoxin does not appear to be limited to anti-microbial and virulence-related activity upon secretion and intracellular gliotoxin appears to play a role in maintaining the redox state of the cell. Owens et al. (2014) demonstrated that gliotoxin alleviates oxidative stress caused by $\mathrm{H}_{2} \mathrm{O}_{2}$ exposure in A. fumigatus which suggests that gliotoxin is not merely a toxin, but may play an important role in redox homeostasis in A. fumigatus. Thus, this SM appears to mediate cross-talk between ROS presence and the global response to ROS in fungi (Figure 1).

Reverberi et al. (2010) have also highlighted a link between production of SMs and oxidative stress. Interestingly, oxidative stress in $A$. parasiticus up-regulates aflatoxin biosynthesis (Jayashree and Subramanyam, 2000; Narasaiah et al., 2006; Reverberi et al., 2008). Conversely, aflatoxin biosynthesis is inhibited in response to antioxidant activity (Reverberi et al., 2005). The aflatoxin regulatory gene, aflR, encodes a $\mathrm{Zn}(\mathrm{II})_{2} \mathrm{Cys}_{6}$ DNA binding protein serves to activate gene expression encoding enzymes for the ST/aflatoxin biosynthetic pathway (Yu et al., 1996). The discovery of putative binding sites for the Yap1 ortholog, AP1, in the aflR promoter region further strengthens this suggested relationship (Reverberi et al., 2008). A similar relationship between oxidative stress and SM production can be seen in Fusarium graminearum. Addition of $\mathrm{H}_{2} \mathrm{O}_{2}$ to liquid cultures increased the accumulation of the type $\mathrm{B}$ trichothecenes, deoxynivalenol, and 15-acetyl-deoxynivalenol, while the addition of catalases down-regulated expression of the genes responsible for their biosynthesis (Ponts et al., 2006, 2007). Similar results showing antioxidants reducing SM biosynthesis have been encountered in A. flavus with aflatoxin (Chipley and Uraih, 1980), F. verticillioides with fumonisin $\mathrm{B}_{1}$ (Beekrum et al., 2003), A. ochraceus with ochratoxin A (Fanelli et al., 2004) and Penicillium expansum with patulin (Sanzani et al., 2009; Tolaini et al., 2010). This demonstrates cross-talk between oxidative stress defense and SM biosynthesis across a range of fungi, producing several apparently unrelated SMs.

Siderophores, the iron-scavenging NRPs described above, have an essential role in oxidative defense. Excess intracellular iron can result in oxidative stress through Fenton reaction (Papanikolaou and Pantopoulos, 2005), while conversely, heme is essential for the function of many peroxidases (Conesa et al., 2002), an important family of enzymes in the detoxification of hydrogen peroxide. Thus it is important for fungi to manage iron levels carefully. Eisendle et al. (2006) demonstrated that oxidative stress causes an up-regulation of the intracellular siderophore ferricrocin in A. nidulans. Such an increase is also seen when exposed to iron excessive conditions, though it is unclear if this is due to the iron itself, or the resulting iron mediated oxidative stress on the cell. 
Deletion of the siderophore synthetase sidC halts production of ferricrocin, resulting in multiple phenotypes, such as inefficient iron utilization, delayed germination in iron-replete conditions and elimination of cleistothecia formation in homothallic conditions. In addition to these phenotypes, the conidia of the sidC mutant display sensitivity to $\mathrm{H}_{2} \mathrm{O}_{2}$. A similar relationship role of siderophores in oxidative defense can be seen in A. fumigatus. Schrettl et al. (2007) investigated several siderophore biosynthetic gene deletions. The siderophore null mutant, $\Delta$ sidA demonstrated severe sensitivity to $\mathrm{H}_{2} \mathrm{O}_{2}$, while absence of either intracellular or extracellular siderophores separately resulted in a milder sensitivity. This suggests that both intra- and extra- cellular siderophores have a role in mediating oxidative damage and suggests redundancy in the system. Cross-talk between ROS detoxification and siderophores is not limited to the Aspergillus species. In Alternaria alternata, deletion of NPS6, a NRPS essential for extracellular siderophore biosynthesis, results in increases sensitivity to $\mathrm{H}_{2} \mathrm{O}_{2}$. In addition, expression of NPS6 in A. alternata is regulated by NOX, YAP1, and HOG1, genes involved in oxidative stress defense (Chen et al., 2014b). Deletion of NPS6 in Cochliobolus miyabeanus, Fusarium graminearum, and Alternaria brassicicola also resulted in increased sensitivity to oxidative stress (Oide et al., 2006).

Medentsev and Akimenko (1998) conducted a large scale investigation into naphthoquinones, redox active SMs produced by a large variety of filamentous fungi. The study demonstrated that many of these (fusarubin, anhydrofusarubin, javanicin, anhydrojavanicin, bostricoidin, norjavanicin, flaviolin, and 2-hydroxyjuglone) displayed activity against bacteria, yeast, fungi, and plant cells. The mode of action of these molecules was through interference with the host respiration system. By accepting the reducing equivalents from redox enzymes and transferring directly to oxygen, resulting in the respiratory chain being bypassed. In addition, naphthoquinones can act through generation of superoxide radicals and inhibition of glutathione reductases.

\section{ENZYME AND CLUSTER CROSS-TALK}

In addition to the clear interactions between primary and secondary metabolism described above, there are also several examples of cross-talk amongst gene clusters which are responsible for the synthesis of fungal SMs (Figure 1). Over-expression of the silent inp putative secondary metabolism cross-pathway regulator gene $(s c p R)$ in $A$. nidulans resulted in the overproduction of both the inp gene cluster and also production of the polyketide, asperfuranone. As no link had previously been described between these unrelated metabolites, this work highlighted some additional complexity of regulatory crosstalk in fungal secondary metabolism (Bergmann et al., 2010). Further recent findings are changing previously held views of distinct SM clusters encoding for single class of, or closely related, SMs. A FAD-dependent monooxygenase (FqzB) which is encoded by the fumiquinazoline biosynthetic pathway in $A$. fumigatus was also shown to catalyze spiro-carbon formation in the indole alkaloid spirotryprostatin A via an epoxidation route. The authors speculated that these interactions between unrelated fungal SM-encoding cluster genes may be a strategy of natural product producers to generate structural diversity (Tsunematsu et al., 2013).

\section{FUMIGACLAVINES AND FUMIQUINAZOLINES}

Fumigaclavine C, an ergot alkaloid, was demonstrated to aid in oxidative stress defense in A. fumigatus whereby deletion of either of the NRP synthetase genes pesL or pes1 resulted in abrogation of the metabolite (O'Hanlon et al., 2012). Loss of fumigaclavine $\mathrm{C}$ in $A$. fumigatus resulted in $\mathrm{H}_{2} \mathrm{O}_{2}$ sensitivity, suggestive of its role in oxidative stress defense. It should be noted that, in contrast to $C$. purpurea (Haarmann et al., 2005), the role of an NRP synthetase in alkaloid synthesis has yet to be confirmed in A. fumigatus. Loss of fumigaclavine C was accompanied by elevated levels of another class of SM, the fumitremorgins. The increased production of fumitremorgins such as TR-2, fumitremorgin $\mathrm{C}$, and verruculogen in response to fumigaclavine $\mathrm{C}$ diminution is indicative of cross-talk within the secondary metabolome. These authors have speculated that increased fumitremorgin levels are possibly due to increased isoprene availability due to decreased prenylation of fumigaclavine A due to PesL/Pes1 absence. This is supported by the findings of Wiemann et al. (2012), who saw an increase in the isoprene derived molecule gibberellin following loss of either PKS or NRPS activity due to deletion of ppt1 in Fusarium fujikuroi. Interestingly, biosynthesis of fumiquinazolines A through $\mathrm{F}$ continues despite the loss of pesL, even though this NRP synthetase has been shown to mediate fumiquinazoline biosynthesis in vitro (Ames et al., 2010), an observation shared by Lim et al. (2014). This suggests that alternative NRP synthetases can be utilized for fumiquinazoline biosynthesis, which implies cross-talk between SM gene clusters.

\section{REDOX CONTROL AND GENE CLUSTER MODULATION}

O'Keeffe et al. (2014) have surprisingly demonstrated that an intact gliotoxin self-protection mechanism (Scharf et al., 2010; Schrettl et al., 2010), mediated by GliT, is essential to regulate the biosynthesis of apparently unrelated metabolites such as pseurotin A, fumagillin, and fumitremorgins. Specifically, using RNA-seq these authors have demonstrated that gene cluster expression encoding the biosynthesis of helvolic acid, pseurotin A, fumagillin and fumitremorgins is significantly attenuated in A. fumigatus $\Delta$ gliT upon gliotoxin exposure. Unexpectedly, alterations in the expression of genes involved in siderophore-mediated iron transport and siderophore biosynthesis was also observed, which was suggestive of a deficiency in iron-sensing. Along with alterations in multiple additional cellular systems, these data suggest that GliT-mediated gliotoxin formation is essential to ensure global SM biosynthesis in A. fumigatus can occur in a regulated manner, and that cellular redox control, amongst other cellular systems, may play an important role in regulating SM biosynthesis (Figure 1).

Further to the observations regarding fumitremorgin and fumigaclavine biosynthesis (O'Hanlon et al., 2012), overexpression of the A. fumigatus putative $\mathrm{Zn}(\mathrm{II})_{2} \mathrm{Cys}_{6}$ transcription factor hasA [which is part of the hexadehydro-astechrome (HAS) biosynthetic gene cluster] also resulted in the production of large amounts of fumitremorgins compared to the wild-type strain. 
Deletion of the HAS NRPS in a background overexpressing has $A$ (OE::has $A$ hasD) resulted in significantly increased fumitremorgin production. The authors hypothesized that the diversion of tryptophan and prenylation activity from HAS production toward fumitremorgin biosynthesis may occur in OE::hasA $\triangle$ hasD due to the inability of this mutant to utilize the pathway intermediates from HAS production. As fumitremorgins are potent tremorgenic mycotoxins this may account for the increased virulence of the OE::hasA $\Delta$ hasD strain compared to wild-type (Yin et al., 2013). This degree of metabolite cross-talk has one limiting affect, because it infers that caution must be exercised in interpreting the impact of metabolite-altering gene deletion experiments. In other words, if metabolite one disappears, and the level of an apparently unrelated metabolite two elevates - which change is altering phenotype?

Although HAS does not contribute to the uptake of extracellular siderophores or iron detoxification in A. fumigatus, an iron starvation phenotype and concomitant siderophore biosynthesis is induced by HAS or astechrome (Wiemann et al., 2014). It has now been demonstrated that iron levels regulate the biosynthesis of HAS, and other SM, which is dependent upon the bZIP protein, HapX and the GATA-type transcription factor, SreA. Wiemann et al. (2014) have also demonstrated that HAS not only influences iron acquisition but also the expression of multiple gene clusters involved in SM biosynthesis. They further speculate that intracellular levels of iron (and HAS) may trigger, at a systems level, primary metabolite availability to effect SM biosynthesis, in a redox-dependent manner. These contemporaneous observations are complementary to those of O'Keeffe et al. (2014) and suggest that we are beginning to piece together the systems interactions, via SM cross-talk, necessary to facilitate SM biosynthesis in this human opportunistic pathogenic fungus (Figure 1).

\section{EFFLUX}

The efflux of SMs is another aspect of secondary metabolism where evidence exists of the occurrence of cross-metabolite interactions. The rice pathogen Magnaporthe grisea produces the PKS-NRPS fusion natural product ACE1 from a 15-gene cluster. Although this cluster encodes a transporter for the MFS superfamily (MFS1), it has been shown that MFS1 is not involved in the efflux of ACE1 as this gene has a deletion of a single base pair which results in an early stop codon. Therefore, it has been suggested that ACE1 must rely on another transporter which is encoded outside of the ACE1 gene cluster (Coleman and Mylonakis, 2009). Moreover, deletion of the sirodesmin $\mathrm{ABC}$ transporter gene sirA from Leptosphaeria maculans actually resulted in an increase (39\%) of sirodesmin production and secretion compared to the wildtype strain. The production of deacetyl sirodesmin in the $\Delta$ sirA mutant also increased $27 \%$ compared to the wild-type. This seemingly contradictory result may be explained by the presence of alternate efflux mechanisms for these metabolites or a degree of redundancy across SM transporters. These alternate efflux mechanisms may be more effective than SirA, resulting in differential sirodesmin cluster feedback regulation which leads to the overexpression of this NRPS cluster (Gardiner et al., 2005b). In contrast, deletion of gliA from the gliotoxin gene cluster significantly decreased gliotoxin efflux, which indicates that some
ETP-producing fungi may not have compensatory mechanisms to mediate natural product efflux (Wang et al., 2014). Evidence also exists to suggest that the MFS transporter DotC encoded within the dothistromin gene cluster of Dothistroma septosporum is not the only mechanism of toxin efflux in this organism (Bradshaw et al., 2009).

\section{INTERTWINED GENE CLUSTERS}

The A. fumigatus genes $p s o F$ (putative dual function methyltransferase and monooxygenase) or psoG (hypothetical protein) were predicted to be required for fumagillin biosynthesis due to their proximity to the characterized fumagillin encoding genes $f m a A$ and $f m a B$. Surprisingly, deletion of $p s o F$ and psoG resulted in the abolition of pseurotin A biosynthesis. The $\triangle p s o F$ strain accumulated a demethyl-deepoxy-synerazol $(\mathrm{m} / \mathrm{z}$ 384.1447) compound instead of pseurotin A which is in agreement with the putative role of this enzyme (Wiemann et al., 2013). PsoF was recently characterized by Tsunematsu et al. (2014). This work highlighted an additional layer of complexity regarding the interactions between SMs and is undoubtedly one of the most dramatic examples of cross-talk between fungal metabolites, at the genetic level, which has been elucidated by Wiemann et al. (2013) in A. fumigatus. Here, the gene clusters encoding fumagillin and the NRP/polyketide hybrid, pseurotin $\mathrm{A}$, are physically intertwined and co-regulated by LaeA via the $\mathrm{Zn}(\mathrm{II})_{2} \mathrm{Cys}_{6}$ Transcription Factor, FapR. In addition, fumitremorgin is also encoded by this supercluster; however, the genes encoding the biosynthesis of this metabolite are distinct from the intertwined region. Interestingly, although this supercluster is not present in completely intact form in related fungal species, there is sufficient co-localization of orthologs to allow Wiemann etal. (2013) to speculate that co-production of the aforementioned metabolites confers survival advantages on producing species. It is tempting to speculate that the products of this supercluster act synergistically or in a complementary manner, almost like subunits of a heteromeric enzyme, to effect survival of $A$. fumigatus, and related species in defined ecological niches.

It is also true to say that hybrid PKS-NRPS megasynthetases represent a unique concept in metabolite cross-talk, whereby PK and NRPs are reconstituted into unique molecular entities and this topic has been extensively reviewed elsewhere (Boettger and Hertweck, 2013).

\section{BEYOND ENDOGENOUS: INTER-KINGDOM CROSS-TALK}

Recent data have also described what may be cross-talk of natural products from different organisms. Culturing A. fumigatus MBC-F1-10 in the presence of Streptomyces bullii leads to the production of a diversity of A. fumigatus metabolites including ergosterol and seven diketopiperazine (DKP) class of alkaloids. Production of the antibiotic-antitumor metabolite glionitrin was induced in A. fumigatus following co-culture with a Sphingomonas isolate KMK-001 derived from mine-drainage system (Rateb et al., 2013). Moreover, physical interaction between the S. rapamycinicus and A. fumigatus resulting in the activation of a silent PKS gene cluster encoding fumicycline A (König et al., 2013). Bacterial metabolites have also been demonstrated to act as precursors of 
fungal metabolites, whereby phenazine metabolites from $\mathrm{Pseu}$ domonas aeruginosa were converted by $A$. fumigatus into new molecular species with enhanced toxicity, as well as additional, properties (Moree et al., 2012). It is likely that further examples of inter-kingdom interactions, which describe cross-talk leading to metabolite production, will be described as increasing attention is being focused on the impacts of co-culturing of microorganisms.

\section{CONCLUDING REMARKS}

Upon writing this manuscript, the authors could not help but be struck once again by two terms which continuously pervade work on fungal natural products - these are NRPS and secondary metabolism. In other words, we ask the question if research in and funding for, this exciting area of chemical biology is hindered by the use of the negative terminology of non and secondary? Perhaps it is time to describe the biosynthesis of these peptidyl entities in more positive ways, and not to refer to the processes of bioactive metabolite formation as a secondary, or somehow less important or optional, system. It is not - evolution generally sees to that! Perhaps CMPS (Cluster Mediated Peptide Synthesis) could replace NRPS, as it simultaneously (i) removes the negative terminology of non, (ii) increases the descriptive value of the acronym to suggest gene cluster involvement in the process, and (iii) offers the possibility of referring to NRPs as or CDPs (Cluster Peptides or Cluster Derived Peptides).

So, it is undoubtedly clear that significant cross-talk exists between (i) primary and secondary metabolism, (ii) different fungal metabolites, (iii) the enzymes involved in the synthesizing different metabolites, and (iv) various metabolite-encoding gene clusters (Figure 1). Indeed cross-talk even exists between kingdoms. As described herein, and elsewhere (Lim and Keller, 2014), the details, significance and potential of this exquisite orchestration of unforeseen molecular events are emerging at an ever-increasing rate. For the future, high-throughput analytical approaches, combined with new insights into, and revised conceptions of, fungal genetics and biochemistry, will yield further surprises which will continue to enthuse us to re-imagine our current perspective of distinct orders of cellular metabolic processes in these truly unique organisms.

\section{ACKNOWLEDGMENTS}

Fungal Research in Sean Doyle's laboratory is funded by a Science Foundation Ireland Principal Investigator Award (SFI/11/PI/1188). Stephen K. Dolan is an Irish Research Council Postgraduate Scholar.

\section{REFERENCES}

Ames, B. D., Liu, X., and Walsh, C. T. (2010). Enzymatic processing of fumiquinazoline F: a tandem oxidative-acylation strategy for the generation of multicyclic scaffolds in fungal indole alkaloid biosynthesis. Biochemistry 49, 8564-8576.

Amich, J., Schafferer, L., Haas, H., and Krappmann, S. (2013). Regulation of sulphur assimilation is essential for virulence and affects iron homeostasis of the human-pathogenic mould Aspergillus fumigatus. PLoS Pathog. 9:e1003573. doi: 10.1371/journal.ppat.1003573

Atanasova, L., Le Crom, S., Gruber, S., Coulpier, F., Seidl-Seiboth, V., Kubicek, C. P., et al. (2013). Comparative transcriptomics reveals different strategies of
Trichoderma mycoparasitism. BMC Genom. 14:121. doi: 10.1186/1471-2164-14121

Balibar, C. J., and Walsh, C. T. (2006). GliP, a multimodular nonribosomal peptide synthetase in Aspergillus fumigatus, makes the diketopiperazine scaffold of gliotoxin. Biochemistry 45, 15029-15038.

Bayram, O., Krappmann, S., Ni, M., Bok, J. W., Helmstaedt, K., Valerius, O., et al. (2008). VelB/VeA/LaeA complex coordinates light signal with fungal development and secondary metabolism. Science 320, 1504-1506. doi: $10.1126 /$ science. 1155888

Beckmann, N., Schafferer, L., Schrettl, M., Binder, U., Talasz, H., Lindner, H., et al. (2013). Characterization of the link between ornithine, arginine, polyamine and siderophore metabolism in Aspergillus fumigatus. PLoS ONE 8:e67426. doi: 10.1371/journal.pone.0067426

Beekrum, S., Govinden, R., Padayachee, T., and Odhav, B. (2003). Naturally occurring phenols: a detoxification strategy for fumonisin B1. Food Addit. Contam. 20, 490-493.

Bello, M. H., Barrera-Perez, V., Morin, D., and Epstein, L. (2012). The Neurospora crassa mutant $\mathrm{Nc} \Delta$ Egt-1 identifies an ergothioneine biosynthetic gene and demonstrates that ergothioneine enhances conidial survival and protects against peroxide toxicity during conidial germination. Fungal Genet. Biol. 49, 160-172. doi: 10.1016/j.fgb.2011.12.007

Bergmann, S., Funk, A. N., Scherlach, K., Schroeckh, V., Shelest, E., Horn, U., et al. (2010). Activation of a silent fungal polyketide biosynthesis pathway through regulatory cross talk with a cryptic nonribosomal peptide synthetase gene cluster. Appl. Environ. Microbiol. 76, 8143-8149. doi: 10.1128/AEM. 00683-10

Bizukojc, M., Pawlowska, B., and Ledakowicz, S. (2007). Supplementation of the cultivation media with B-group vitamins enhances lovastatin biosynthesis by aspergillus terreus. J. Biotechnol. 127, 258-268. doi: 10.1016/j.jbiotec.2006. 06.017

Boettger, D., and Hertweck, C. (2013). Molecular diversity sculpted by fungal PKSNRPS hybrids. ChemBioChem 14, 28-42.

Bok, J. W., and Keller, N. P. (2004). LaeA, a regulator of secondary metabolism in aspergillus spp. Eukaryot. Cell 3, 527-535.

Bradshaw, R. E., Feng, Z., Schwelm, A., Yang, Y., and Zhang, S. (2009). Functional analysis of a putative dothistromin toxin MFS transporter gene. Toxins 1 , 173-187.

Carlsson, J., Kierstan, M. P., and Brocklehurst, K. (1974). Reactions of Lergothioneine and some other aminothiones with2,2'-and 4,4'-dipyridyl disulphides and of L-ergothioneine with iodoacetamide. 2-mercaptoimidazoles, 2- and 4-thiopyridones, thiourea and thioacetamide as highly reactive neutral sulphur nucleophils. Biochem. J. 139, 221-235.

Chen, H., He, X., Geng, H., and Liu, H. (2014a). Physiological characterization of ATP-citrate lyase in aspergillus niger. J. Ind. Microbiol. Biotechnol. 41, 721-731.

Chen, L. H., Yang, S. L., and Chung, K. R. (2014b). Resistance to oxidative stress via regulating siderophore-mediated iron acquisition by the citrus fungal pathogen Alternaria alternata. Microbiology 160(Pt 5), 970-979. doi: 10.1099/mic.0.076182-0

Chipley, J. R., and Uraih, N. (1980). Inhibition of aspergillus growth and aflatoxin release by derivatives of benzoic acid. Appl. Environ. Microbiol. 40, 352-357.

Chocklett, S. W., and Sobrado, P. (2010). Aspergillus fumigatus SidA is a highly specific ornithine hydroxylase with bound flavin cofactor. Biochemistry 49, 67776783.

Chujo, T., and Scott, B. (2014). Histone H3K9 and H3K27 methylation regulates fungal alkaloid biosynthesis in a fungal endophyte-plant symbiosis. Mol. Microbiol. 92, 413-34. doi: 10.1111/mmi.12567

Coleman, J. J., and Mylonakis, E. (2009). Efflux in fungi: la piece de resistance. PLoS Pathog. 5:e1000486. doi: 10.1371/journal.ppat.1000486

Conesa, A., Punt, P. J., and van den Hondel, C. A. (2002). Fungal peroxidases: molecular aspects and applications. J. Biotechnol. 93, 143-158.

Davis, C., Carberry, S., Schrettl, M., Singh, I., Stephens, J. C., Barry, S. M., et al. (2011). The role of glutathione S-transferase GliG in gliotoxin biosynthesis in Aspergillus fumigatus. Chem. Biol. 18, 542-552.

Dolan, S. K., Owens, R. A., O’Keeffe, G., Hammel, S., Fitzpatrick, D. A., Jones, G. W., et al. (2014). Regulation of nonribosomal peptide synthesis: bis-thiomethylation attenuates gliotoxin biosynthesis in Aspergillus fumigatus. Chem. Biol. 21, 9991012 . 
Eisendle, M., Schrettl, M., Kragl, C., Muller, D., Illmer, P., and Haas, H. (2006). The intracellular siderophore ferricrocin is involved in iron storage, oxidativestress resistance, germination, and sexual development in Aspergillus nidulans. Eukaryot. Cell 5, 1596-1603.

Elliott, C. E., Fox, E. M., Jarvis, R. S., and Howlett, B. J. (2011). The crosspathway control system regulates production of the secondary metabolite toxin sirodesmin PL, in the ascomycete, Leptosphaeria maculans. BMC Microbiol. 11:169. doi: 10.1186/1471-2180-11-169

Fahey, R. C. (2013). Glutathione analogs in prokaryotes. Biochim. Biophys. Acta 1830, 3182-3198.

Fan, J., Ye, J., Kamphorst, J. J., Shlomi, T., Thompson, C. B., and Rabinowitz, J. D. (2014). Quantitative flux analysis reveals folate-dependent NADPH production. Nature 510, 298-302. doi: 10.1038/nature13236

Fanelli, C., Ricelli, A., Reverberi, M., Fabbri, A., and Pandalai, S. (2004). Aflatoxins and ochratoxins in cereal grains: an open challenge. Recent Res. Dev. Crop Sci. 1, 295-317.

Forseth, R. R., Fox, E. M., Chung, D., Howlett, B. J., Keller, N. P., and Schroeder F. C. (2011). Identification of cryptic products of the gliotoxin gene cluster using NMR-based comparative metabolomics and a model for gliotoxin biosynthesis. J. Am. Chem. Soc. 133, 9678-9681.

Franken, A. C., Lechner, B. E., Werner, E. R., Haas, H., Lokman, B. C., Ram, A. F., et al. (2014). Genome mining and functional genomics for siderophore production in aspergillus niger. Brief. Funct. Genom. 13, 482-492.

Gallagher, L., Owens, R. A., Dolan, S. K., O'Keeffe, G., Schrettl, M., Kavanagh, K., et al. (2012). The Aspergillus fumigatus protein GliK protects against oxidative stress and is essential for gliotoxin biosynthesis. Eukaryot. Cell 11, 1226-1238. doi: 10.1128/EC.00113-12

Gardiner, D. M., Waring, P., and Howlett, B. J. (2005a). The epipolythiodioxopiperazine (ETP) class of fungal toxins: distribution, mode of action, functions and biosynthesis. Microbiology 151(Pt 4), 1021-1032.

Gardiner, D. M., Jarvis, R. S., and Howlett, B. J. (2005b). The ABC transporter gene in the sirodesmin biosynthetic gene cluster of Leptosphaeria maculans is not essential for sirodesmin production but facilitates self-protection. Fungal Genet. Biol. 42, 257-263.

Genghof, D. S. (1970). Biosynthesis of ergothioneine and hercynine by fungi and actinomycetales. J. Bacteriol. 103, 475-478.

Gerke, J., Bayram, Ö., and Braus, G. H. (2012). Fungal S-adenosylmethionine synthetase and the control of development and secondary metabolism in Aspergillus nidulans. Fungal Genet. Biol. 49, 443-454.

Griffiths, E. J., Hu, G., Fries, B., Caza, M., Wang, J., Gsponer, J., et al. (2012). A defect in ATP-citrate lyase links acetyl-CoA production, virulence factor elaboration and virulence in Cryptococcus neoformans. Mol. Microbiol. 86, 1404-1423.

Guo, C., Knox, B. P., Sanchez, J. F., Chiang, Y., Bruno, K. S., and Wang, C. C. (2013a). Application of an efficient gene targeting system linking secondary metabolites to their biosynthetic genes in Aspergillus terreus. Org. Lett. 15, 3562-3565.

Guo, C., Yeh, H., Chiang, Y., Sanchez, J. F., Chang, S., Bruno, K. S., et al. (2013b). Biosynthetic pathway for the epipolythiodioxopiperazine acetylaranotin in Aspergillus terreus revealed by genome-based deletion analysis. J. Am. Chem. Soc. 135, 7205-7213.

Haarmann, T., Machado, C., Lübbe, Y., Correia, T., Schardl, C. L., Panaccione, D. G., et al. (2005). The ergot alkaloid gene cluster in Claviceps purpurea: extension of the cluster sequence and intra species evolution. Phytochemistry 66, 1312-1320.

Haas, H. (2012). Iron - A key nexus in the virulence of Aspergillus fumigatus. Front. Microbiol. 3:28. doi: 10.3389/fmicb.2012.00028

Halliwell, B., and Gutteridge, J. (2007). "Cellular responses to oxidative stress: adaptation, damage, repair, senescence and death," in Free Radicals in Biology and Medicine, 4th Edn, eds B. Halliwell and J. M. C. Gutteridge (New York: Oxford University Press), 187-267.

Hansen, F. T., Droce, A., Sørensen, J. L., Fojan, P., Giese, H., and Sondergaard, T. E. (2012). Overexpression of NRPS4 leads to increased surface hydrophobicity in Fusarium graminearum. Fungal Biol. 116, 855-862.

Hissen, A. H., Wan, A. N., Warwas, M. L., Pinto, L. J., and Moore, M. M. (2005). The Aspergillus fumigatus siderophore biosynthetic gene sidA, encoding L-ornithine N5-oxygenase, is required for virulence. Infect. Immun. 73, 5493-5503.

Jayashree, T., and Subramanyam, C. (2000). Oxidative stress as a prerequisite for aflatoxin production by aspergillus parasiticus. Free Radic. Biol. Med. 29, 981-985. doi: 10.1016/S0891-5849(00)00398-1
Jeon, J. H., and Lee, Y. H. (2014). Histone acetylation in fungal pathogens of plants. Plant Pathol. J. 30, 1-9.

Jocelyn, P. (1972). Biochemistry of the SH Group: The Occurrence, Chemical Properties, Metabolism and Biological Functions of Thiols and Disulfides. London, NY: Academic Press.

Jones, G. W., Doyle, S., and Fitzpatrick, D. A. (2014). The evolutionary history of the genes involved in the biosynthesis of the antioxidant ergothioneine. Gene 549, 161-170.

König, C. C., Scherlach, K., Schroeckh, V., Horn, F., Nietzsche, S., Brakhage, A. A., etal. (2013). Bacterium induces cryptic meroterpenoid pathway in the pathogenic fungus Aspergillus fumigatus. ChemBioChem 14, 938-942.

Leal, S. M. Jr., Roy, S., Vareechon, C., deJesus Carrion, S., Clark, H., LopezBerges, M. S., et al. (2013). Targeting iron acquisition blocks infection with the fungal pathogens Aspergillus fumigatus and Fusarium oxysporum. PLoS Pathog. 9:e1003436. doi: 10.1371/journal.ppat.1003436

Li, M., Kim, T.-J., Kwon, H.-J., and Suh, J.-W. (2008). Effects of extracellular ATP on the physiology of Streptomyces coelicolor A3(2). FEMS Microbiol. Lett. 286, 24-31. doi: 10.1111/j.1574-6968.2008.01248.x

Liao, S., Li, R., Shi, L., Wang, J., Shang, J., Zhu, P., et al. (2012). Functional analysis of an S-adenosylhomocysteine hydrolase homolog of chestnut blight fungus. FEMS Microbiol. Lett. 336, 64-72.

Lim, F. Y., Ames, B., Walsh, C. T., and Keller, N. P. (2014). Co-ordination between BrlA regulation and secretion of the oxidoreductase FmqD directs selective accumulation of fumiquinazoline $\mathrm{C}$ to conidial tissues in Aspergillus fumigatus. Cell. Microbiol. 16, 1267-1283. doi: 10.1111/cmi.12284

Lim, F. Y., and Keller, N. P. (2014). Spatial and temporal control of fungal natural product synthesis. Nat. Prod. Rep. 31, 1277-1286.

Lushchak, V. I. (2011). Adaptive response to oxidative stress: bacteria, fungi, plants and animals. Comp. Biochem. Physiol. C Toxicol. Pharmacol. 153, 175-190.

Manavalan, B., Murugapiran, S. K., Lee, G., and Choi, S. (2010). Molecular modeling of the reductase domain to elucidate the reaction mechanism of reduction of peptidyl thioester into its corresponding alcohol in non-ribosomal peptide synthetases. BMC Struct. Biol. 10:1. doi: 10.1186/1472-6807-10-1

McDonagh, A., Fedorova, N. D., Crabtree, J., Yu, Y., Kim, S., Chen, D., et al. (2008). Sub-telomere directed gene expression during initiation of invasive aspergillosis. PLoS Pathog. 4:e1000154. doi: 10.1371/journal.ppat.1000154

Medentsev, A., and Akimenko, V. (1998). Naphthoquinone metabolites of the fungi. Phytochemistry 47, 935-959.

Meier, J. L., and Burkart, M. D. (2009). The chemical biology of modular biosynthetic enzymes. Chem. Soc. Rev. 38, 2012-2045.

Middleton, M. C. (1974). The involvement of the disulphide group of sporidesmin in the action of the toxin on swelling and respiration of liver mitochondria. Biochem. Pharmacol. 23, 811-820.

Moree, W. J., Phelan, V. V., Wu, C. H., Bandeira, N., Cornett, D. S., Duggan, B. M., et al. (2012). Interkingdom metabolic transformations captured by microbial imaging mass spectrometry. Proc. Natl. Acad. Sci. U.S.A. 109, 13811-13816. doi: 10.1073/pnas. 1206855109

Munday, R. (1982). Studies on the mechanism of toxicity of the mycotoxin, sporidesmin. I. generation of superoxide radical by sporidesmin. Chem. Biol. Interact. 41, 361-374.

Narasaiah, K. V., Sashidhar, R., and Subramanyam, C. (2006). Biochemical analysis of oxidative stress in the production of aflatoxin and its precursor intermediates. Mycopathologia 162, 179-189.

Niehaus, E.-M., Janevska, S., von Bargen, K. W., Sieber, C. M. K., Harrer, H., Humpf, H.-U., et al. (2014). Apicidin F: characterization and genetic manipulation of a new secondary metabolite gene cluster in the rice pathogen Fusarium fujikuroi. PLoS ONE 9:e103336. doi: 10.1371/journal.pone.0103336

O’Hanlon, K. A., Cairns, T., Stack, D., Schrettl, M., Bignell, E. M., Kavanagh, K., et al. (2011). Targeted disruption of nonribosomal peptide synthetase pes3 augments the virulence of Aspergillus fumigatus. Infect. Immun. 79, 3978-3992. doi: 10.1128/IAI.00192-11

O'Hanlon, K. A., Gallagher, L., Schrettl, M., Jochl, C., Kavanagh, K., Larsen, T. O., et al. (2012). Nonribosomal peptide synthetase genes pesL and pes1 are essential for fumigaclavine C production in Aspergillus fumigatus. Appl. Environ. Microbiol. 78, 3166-3176. doi: 10.1128/AEM.07249-11

Oide, S., Moeder, W., Krasnoff, S., Gibson, D., Haas, H., Yoshioka, K., et al. (2006). NPS6, encoding a nonribosomal peptide synthetase involved in siderophore- 
mediated iron metabolism, is a conserved virulence determinant of plant pathogenic ascomycetes. Plant Cell 18, 2836-2853.

O’Keeffe, G., Hammel, S., Owens, R. A., Keane, T. M., Fitzpatrick, D. A., Jones, G. W., et al. (2014). RNA-seq reveals the pan-transcriptomic impact of attenuating the gliotoxin self-protection mechanism in Aspergillus fumigatus. BMC Genom. 15:894. doi: 10.1186/1471-2164-15-894

Owens, R. A., Hammel, S., Sheridan, K. J., Jones, G. W., and Doyle, S. (2014) A proteomic approach to investigating gene cluster expression and secondary metabolite functionality in Aspergillus fumigatus. PLOS ONE 9:e106942. doi: 10.1371/journal.pone.0106942

Papanikolaou, G., and Pantopoulos, K. (2005). Iron metabolism and toxicity. Toxicol. Appl. Pharmacol. 202, 199-211.

Pluskal, T., Ueno, M., and Yanagida, M. (2014). Genetic and metabolomic dissection of the ergothioneine and selenoneine biosynthetic pathway in the fission yeast, $\mathrm{S}$. pombe, and construction of an overproduction system. PLOS ONE 9:e97774. doi: 10.1371/journal.pone.0097774

Ponts, N., Pinson-Gadais, L., Barreau, C., Richard-Forget, F., and Ouellet, T. (2007). Exogenous $\mathrm{H} 2 \mathrm{O} 2$ and catalase treatments interfere with tri genes expression in liquid cultures of Fusarium graminearum. FEBS Lett. 581, 443-447. doi: 10.1016/j.febslet.2007.01.003

Ponts, N., Pinson-Gadais, L., Verdal-Bonnin, M., Barreau, C., and Richard-Forget, F. (2006). Accumulation of deoxynivalenol and its 15 -acetylated form is significantly modulated by oxidative stress in liquid cultures of Fusarium graminearum. FEMS Microbiol. Lett. 258, 102-107.

R Poulsen, B., Nøhr, J., Douthwaite, S., Hansen, L. V., Iversen, J. J., Visser, J., et al. (2005). Increased NADPH concentration obtained by metabolic engineering of the pentose phosphate pathway in Aspergillus niger. FEBS J. 272, 1313-1325.

Rateb, M. E., Hallyburton, I., Houssen, W. E., Bull, A. T., Goodfellow, M., Santhanam, R., et al. (2013). Induction of diverse secondary metabolites in Aspergillus fumigatus by microbial co-culture. RSC Adv. 3, 14444-14450.

Reverberi, M., Fabbri, A., Zjalic, S., Ricelli, A., Punelli, F., and Fanelli, C. (2005) Antioxidant enzymes stimulation in Aspergillus parasiticus by Lentinula edodes inhibits aflatoxin production. Appl. Microbiol. Biotechnol. 69, 207-215.

Reverberi, M., Ricelli, A., Zjalic, S., Fabbri, A. A., and Fanelli, C. (2010). Natural functions of mycotoxins and control of their biosynthesis in fungi. Appl Microbiol. Biotechnol. 87, 899-911.

Reverberi, M., Zjalic, S., Ricelli, A., Punelli, F., Camera, E., Fabbri, C., et al. (2008) Modulation of antioxidant defense in Aspergillus parasiticus is involved in aflatoxin biosynthesis: a role for the ApyapA gene. Eukaryot. Cell 7, 988-1000. doi: 10.1128/EC.00228-07

Reyes-Dominguez, Y., Bok, J. W., Berger, H., Shwab, E. K., Basheer, A., Gallmetzer, A., et al. (2010). Heterochromatic marks are associated with the repression of secondary metabolism clusters in Aspergillus nidulans. Mol. Microbiol. 76, 13761386. doi: 10.1111/j.1365-2958.2010.07051.x

Sanzani, S. M., Schena, L., Nigro, F., De Girolamo, A., and Ippolito, A. (2009). Effect of quercetin and umbelliferone on the transcript level of Penicillium expansum genes involved in patulin biosynthesis. Eur. J. Plant Pathol. 125, 223-233.

Sauter, M., Moffatt, B., Saechao, M. C., Hell, R., and Wirtz, M. (2013). Methionine salvage and S-adenosylmethionine: essential links between sulfur, ethylene and polyamine biosynthesis. Biochem. J. 451, 145-154.

Scharf, D. H., Chankhamjon, P., Scherlach, K., Heinekamp, T., Willing, K., Brakhage, A. A., et al. (2013). Epidithiodiketopiperazine biosynthesis: a four-enzyme cascade converts glutathione conjugates into transannular disulfide bridges. Angew. Chem. Int. Ed. 52, 11092-11095.

Scharf, D. H., Habel, A., Heinekamp, T., Brakhage, A. A., and Hertweck, C. (2014). Opposed effects of enzymatic gliotoxin N-and S-methylations. J. Am. Chem. Soc. 136, 11674-11679.

Scharf, D. H., Remme, N., Habel, A., Chankhamjon, P., Scherlach, K., Heinekamp, T., et al. (2011). A dedicated glutathione S-transferase mediates carbon-sulfur bond formation in gliotoxin biosynthesis. J. Am. Chem. Soc. 133, 12322-12325.

Scharf, D. H., Remme, N., Heinekamp, T., Hortschansky, P., Brakhage, A. A., and Hertweck, C. (2010). Transannular disulfide formation in gliotoxin biosynthesis and its role in self-resistance of the human pathogen Aspergillus fumigatus. J. Am. Chem. Soc. 132, 10136-10141.

Schrettl, M., Bignell, E., Kragl, C., Joechl, C., Rogers, T., Arst, H. N., et al. (2004). Siderophore biosynthesis but not reductive iron assimilation is essential for Aspergillus fumigatus virulence. J. Exp. Med. 200, 1213-1219.
Schrettl, M., Bignell, E., Kragl, C., Sabiha, Y., Loss, O., Eisendle, M., et al. (2007). Distinct roles for intra-and extracellular siderophores during Aspergillus fumigatus infection. PLoS Pathog. 3:e128. doi: 10.1371/journal.ppat.0030128

Schrettl, M., Carberry, S., Kavanagh, K., Haas, H., Jones, G. W., O’Brien, J., et al. (2010). Self-protection against gliotoxin-a component of the gliotoxin biosynthetic cluster, GliT, completely protects Aspergillus fumigatus against exogenous gliotoxin. PLoS Pathog. 6:e1000952. doi: 10.1371/journal.ppat. 1000952

Seebeck, F. P. (2010). In vitro reconstitution of mycobacterial ergothioneine biosynthesis. J. Am. Chem. Soc. 132, 6632-6633.

Shwab, E. K., Bok, J. W., Tribus, M., Galehr, J., Graessle, S., and Keller, N. P. (2007). Histone deacetylase activity regulates chemical diversity in Aspergillus. Eukaryot. Cell 6, 1656-64. doi: 10.1128/EC.00186-07

Sieńko, M., Natorff, R., Owczarek, S., Olewiecki, I., and Paszewski, A. (2009). Aspergillus nidulans genes encoding reverse transsulfuration enzymes belong to homocysteine regulon. Curr. Genet. 55, 561-570.

Spry, C., Kirk, K., and Saliba, K. J. (2008). Coenzyme A biosynthesis: an antimicrobial drug target. FEMS Microbiol. Rev. 32, 56-106.

Stack, D., Frizzell, A., Tomkins, K., and Doyle, S. (2009). Solid phase 4'phosphopantetheinylation: fungal thiolation domains are targets for chemoenzymatic modification. Bioconjug. Chem. 20, 1514-1522.

Stack, D., Neville, C., and Doyle, S. (2007). Nonribosomal peptide synthesis in Aspergillus fumigatus and other fungi. Microbiology 153(Pt 5), 1297-1306.

Starkov, A. A. (2008). The role of mitochondria in reactive oxygen species metabolism and signaling. Ann. N. Y. Acad. Sci. 1147, 37-52.

Steinchen, W., Lackner, G., Yasmin, S., Schrettl, M., Dahse, H. M., Haas, H., et al. (2013). Bimodular peptide synthetase SidE produces fumarylalanine in the human pathogen Aspergillus fumigatus. Appl. Environ. Microbiol. 79, 6670-6676. doi: 10.1128/AEM.02642-13

Takemoto, D., Tanaka, A., and Scott, B. (2007). NADPH oxidases in fungi: diverse roles of reactive oxygen species in fungal cellular differentiation. Fungal Genet. Biol. 44, 1065-1076.

Thykaer, J., and Nielsen, J. (2003). Metabolic engineering of $\beta$-lactam production. Metab. Eng. 5, 56-69.

Tolaini, V., Zjalic, S., Reverberi, M., Fanelli, C., Fabbri, A. A., Del Fiore, A., et al. (2010). Lentinula edodes enhances the biocontrol activity of Cryptococcus laurentii against Penicillium expansum contamination and patulin production in apple fruits. Int. J. Food Microbiol. 138, 243-249. doi: 10.1016/j.ijfoodmicro.2010. 01.044

Tsunematsu, Y., Fukutomi, M., Saruwatari, T., Noguchi, H., Hotta, K., Tang, Y., et al. (2014). Elucidation of pseurotin biosynthetic pathway points to Trans-Acting CMethyltransferase: generation of chemical diversity. Angew. Chem. Int. Ed. 53, 8475-8479.

Tsunematsu, Y., Ishikawa, N., Wakana, D., Goda, Y., Noguchi, H., Moriya, H., et al. (2013). Distinct mechanisms for spiro-carbon formation reveal biosynthetic pathway crosstalk. Nat. Chem. Biol. 9, 818-825. doi: 10.1038/nchembio.1366

Umemura, M., Nagano, N., Koike, H., Kawano, J., Ishii, T., Miyamura, Y., et al. (2014). Characterization of the biosynthetic gene cluster for the ribosomally synthesized cyclic peptide ustiloxin B in Aspergillus flavus. Fungal Genet. Biol. 68, $23-30$.

Wackler, B., Lackner, G., Chooi, Y. H., and Hoffmeister, D. (2012). Characterization of the Suillus grevillei quinone synthetase GreA supports a nonribosomal code for aromatic $\alpha$-keto acids. Chembiochem 13, 1798-1804. doi: 10.1002/cbic.201200187

Walsh, C. T., Haynes, S. W., Ames, B. D., Gao, X., and Tang, Y. (2013). Short pathways to complexity generation: fungal peptidyl alkaloid multicyclic scaffolds from anthranilate building blocks. ACS Chem. Biol. 8, 1366-1382. doi: $10.1021 / \mathrm{cb} 4001684$

Wang, D. N., Toyotome, T., Muraosa, Y., Watanabe, A., Wuren, T., Bunsupa, S., et al. (2014). GliA in Aspergillus fumigatus is required for its tolerance to gliotoxin and affects the amount of extracellular and intracellular gliotoxin. Med. Mycol. 52, 506-518. doi: 10.1093/mmy/myu007

Waring, P., Sjaarda, A., and Lin, Q. H. (1995). Gliotoxin inactivates alcohol dehydrogenase by either covalent modification or free radical damage mediated by redox cycling. Biochem. Pharmacol. 49, 1195-1201.

Weissman, K. J. (2014). Uncovering the structures of modular polyketide synthases. Nat. Prod. Rep. doi: 10.1039/C4NP00098F [Epub ahead of print].

Wiemann, P., Albermann, S., Niehaus, E., Studt, L., Von Bargen, K. W., Brock, N. L., et al. (2012). The sfp-type 4'-phosphopantetheinyl transferase Pptl of Fusarium 
fujikuroi controls development, secondary metabolism and pathogenicity. PLoS ONE 7:e37519. doi: 10.1371/journal.pone.0037519

Wiemann, P., Brown, D. W., Kleigrewe, K., Bok, J. W., Keller, N. P., Humpf, H.U., et al. (2010). FfVell and FfLael, components of a velvet-like complex in Fusarium fujikuroi, affect differentiation, secondary metabolism and virulence. Mol. Microbiol. doi: 10.1111/j.1365-2958.2010.07263.x [Epub ahead of print].

Wiemann, P., Guo, C. J., Palmer, J. M., Sekonyela, R., Wang, C. C., and Keller, N. P. (2013). Prototype of an intertwined secondary-metabolite supercluster. Proc. Natl. Acad. Sci. U.S.A. 110, 17065-17070. doi: 10.1073/pnas.1313258110

Wiemann, P., and Keller, N. P. (2014). Strategies for mining fungal natural products. J. Ind. Microbiol. Biotechnol. 41, 301-313.

Wiemann, P., Lechner, B. E., Baccile, J. A., Velk, T. A., Yin, W.-B., Bok, J. W., et al. (2014). Perturbations in small molecule synthesis uncovers an iron-responsive secondary metabolite network in Aspergillus fumigatus. Front. Microbiol. 5:530 doi: 10.3389/fmicb.2014.00530

Wilson, D. J., Shi, C., Teitelbaum, A. M., Gulick, A. M., and Aldrich, C. C. (2013). Characterization of AusA: a dimodular nonribosomal peptide synthetase responsible for the production of aureusimine pyrazinones. Biochemistry 52, 926-937.

Yasmin, S., Alcazar-Fuoli, L., Grundlinger, M., Puempel, T., Cairns, T., Blatzer, M., et al. (2012). Mevalonate governs interdependency of ergosterol and siderophore biosyntheses in the fungal pathogen Aspergillus fumigatus. Proc. Natl. Acad. Sci. U.S.A. 109, E497-E504. doi: 10.1073/pnas.1106399108

Yin, W., Baccile, J. A., Bok, J. W., Chen, Y., Keller, N. P., and Schroeder, F. C. (2013). A nonribosomal peptide synthetase-derived iron (III) complex from the pathogenic fungus Aspergillus fumigatus. J. Am. Chem. Soc. 135, 2064-2067.
Yu, J. H., Butchko, R. A., Fernandes, M., Keller, N. P., Leonard, T. J., and Adams, T. H. (1996). Conservation of structure and function of the aflatoxin regulatory gene aflR from Aspergillus nidulans and A. flavus. Curr. Genet. 29, 549-555.

Zhang, N., NurAinIzzati, M. Z., Scher, K., Condon, B. J., Horwitz, B. A., and Turgeon, B. G. (2013). Iron, oxidative stress, and virulence: roles of iron-sensitive transcription factor Srel and the redox sensor ChApl in the maize pathogen Cochliobolus heterostrophus. Mol. Plant Microbe Interact. 26, 1473-1485.

Conflict of Interest Statement: The authors declare that the research was conducted in the absence of any commercial or financial relationships that could be construed as a potential conflict of interest.

Received: 31 October 2014; accepted: 04 December 2014; published online: 05 January 2015.

Citation: Sheridan KJ, Dolan SK and Doyle S (2015) Endogenous cross-talk of fungal metabolites. Front. Microbiol. 5:732. doi: 10.3389/fmicb.2014.00732

This article was submitted to Microbial Physiology and Metabolism, a section of the journal Frontiers in Microbiology.

Copyright (c) 2015 Sheridan, Dolan and Doyle. This is an open-access article distributed under the terms of the Creative Commons Attribution License (CC BY). The use, distribution or reproduction in other forums is permitted, provided the original author(s) or licensor are credited and that the original publication in this journal is cited, in accordance with accepted academic practice. No use, distribution or reproduction is permitted which does not comply with these terms. 